\title{
Language Neuroplasticity in Brain Tumor Patients Revealed by Magnetoencephalography
}

\author{
Vitória Piai $^{1,2}$, Elke De Witte ${ }^{3,4}$, Joanna Sierpowska ${ }^{1,2}$, Xiaochen Zheng $^{1}$ \\ Leighton B. Hinkley ${ }^{4}$, Danielle Mizuiri ${ }^{4}$, Robert T. Knight ${ }^{5}$ \\ Mitchel S. Berger ${ }^{4}$, and Srikantan S. Nagarajan ${ }^{4}$
}

\begin{abstract}
Little is known about language impairment in brain tumor patients, especially in the presurgical phase. Impairment in this population may be missed because standardized tests fail to capture mild deficits. Additionally, neuroplasticity may also contribute to minimizing language impairments. We examined 14 presurgical patients with brain tumors in the languagedominant hemisphere using magnetoencephalography (MEG) while they performed a demanding picture-word interference task, that is, participants name pictures while ignoring distractor words. Brain tumor patients had behavioral picture-naming effects typically observed in healthy controls. The MEG responses also showed the expected pattern in its timing and amplitude modulation typical of controls, but with an altered spatial distribution of right hemisphere sources, in contrast to the classic left hemisphere source found in healthy individuals. This
\end{abstract}

\section{INTRODUCTION}

Brain tumor surgery aims to prolong survival by removing pathological tissue while avoiding deficits (Duffau, 2007). This approach requires the use of tests that are sensitive enough to capture subtle impairment (Brownsett et al., 2019; De Witte et al., 2015; see also Sierpowska et al., 2017; Rofes \& Miceli, 2014). Protocols often make use of standard neuropsychological tests, which are sensitive to impairments in the moderate-to-severe range, such as those seen in stroke-induced aphasia. However, in the brain tumor population, subtle language and cognitive impairment may go unnoticed before surgery because standardized tests may fail to capture mild deficits (Satoer, Vincent, Smits, Dirven, \& Visch-brink, 2013). Additionally, brain plasticity may occur presurgically (Duffau, 2014), mitigating language impairment.

\footnotetext{
${ }^{1}$ Radboud University, Nijmegen, the Netherlands, ${ }^{2}$ Radboud University Medical Center, Nijmegen, the Netherlands, ${ }^{3}$ Free University of Brussels, ${ }^{4}$ University of California, San Francisco, ${ }^{5}$ University of California, Berkeley
}

finding supports tumor-induced neural reorganization of language before surgery. Crucially, the use of electrophysiology allowed us to show the "same" neuronal response in terms of its timing and amplitude modulation in the right hemisphere, supporting the hypothesis that the processes performed by the right hemisphere following reorganization are similar in nature to those (previously) performed by the left hemisphere. We also identified one participant with a fast-growing tumor affecting large parts of critical language areas and underlying ventral and dorsal white matter tracts who showed a deviant pattern in behavior and in the MEG event-related responses. In conclusion, our results attest to the validity of using a demanding picturenaming task in presurgical patients and provide evidence for neuroplasticity, with the right hemisphere performing similar computations as the left hemisphere typically performs.
There is limited information on language impairment in brain tumor patients and on the relationship between impairment and lesion location (Satoer, Visch-Brink, Dirven, \& Vincent, 2016), especially in the presurgical phase. This study examines presurgical brain tumor patients performing an attentional demanding picturenaming task while their brain activity was monitored with magnetoencephalography (MEG). Speaking is an attentionally demanding task (Roelofs \& Piai, 2011), and the picture-word interference paradigm is sensitive to the attentional control demands necessary for naming a picture while ignoring distracting information (Piai \& Knight, 2018; Piai, Riès, \& Swick, 2016). In this paradigm, participants are asked to name a picture displayed on the screen while ignoring a distractor word, presented either auditorily or in written form superimposed onto the picture (see Figure 1 for an example). Previous studies have found that semantic interference (more difficult picture naming with categorically related distractors than with unrelated distractors; see Figure 1) implicates the left temporal lobe (Piai \& Knight, 2018; Piai, Roelofs, Jensen, Schoffelen, \& Bonnefond, 2014). By contrast, lexical interference (more difficult picture 


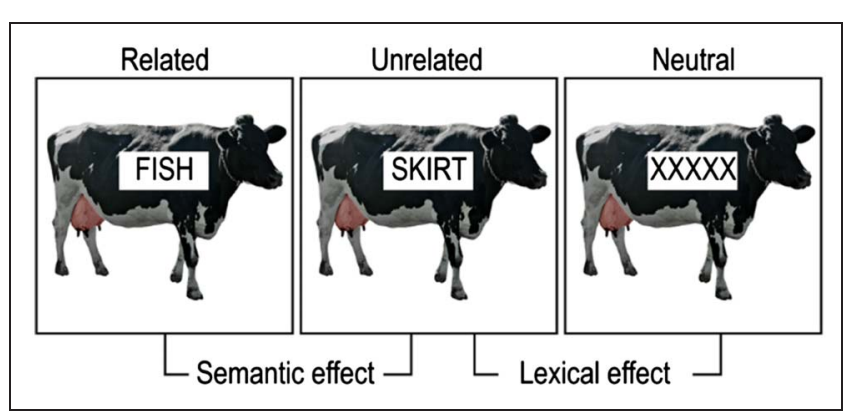

Figure 1. Picture-word interference task. Example of picture-word interference stimuli for related (left), unrelated (middle), and neutral (right) distractors and the corresponding interference effects.

naming with lexical distractors than with a neutral XXX string; see Figure 1) implicates the ventrolateral prefrontal cortex (Piai et al., 2016). Brain tumors often grow along white matter pathways in perisylvian language-related areas (Anderson, Damasio, \& Tranel, 1990), so the temporal lobe and the ventrolateral prefrontal cortex are cortical terminations whose functions may be affected because of the tumor.

MEG provides a direct measure of neuronal activity in the subsecond timescale with enhanced localization capacity. Certain neuronal "signatures" are well characterized in the literature in terms of their timing, associated brain areas, and sensitivity to experimental manipulations. These signatures may enable a better understanding of neuroplasticity, as one can examine whether a certain signature typically found in a left hemisphere brain area is now reorganized in a brain-lesioned individual (e.g., Traut et al., 2019; Piai, Meyer, Dronkers, \& Knight, 2017). There is a robust neurophysiological signature of lexical-semantic processing in picture naming, expressed as an amplitude modulation of the N400 event-related component (de Zubicaray \& Piai, 2019). The N400 is an ERP that peaks approximately 400 msec poststimulus onset and has multiple sources in the left temporal cortex (Lau, Phillips, \& Poeppel, 2008). In picture-word interference, enhanced N400 responses are found for unrelated relative to related picture-distractor pairs in the left temporal cortex (de Zubicaray \& Piai, 2019; Piai et al., 2014).

The present study had several aims. First, we assessed the feasibility of administering picture-word interference, an attentionally demanding task, in presurgical brain tumor patients while recording their brain activity at the subsecond timescale using MEG. More importantly, we aimed to identify any deficits in word production in the presurgical phase and examine patterns of neural reorganization due to tumors. For that, we focused on the MEG counterpart of the N400 component, the N400m, as a functional measure of lexical-semantic processes. Given that this event-related response has a well-known spatiotemporal characterization (de Zubicaray \& Piai, 2019; Kutas \& Federmeier, 2011), any changes in its timing or spatial components would support neuronal reorganization of language functioning.

\section{METHODS}

\section{Participants}

Fourteen consecutive individuals (eight women; mean age at testing $=42.4$ years) with tumors in the languagedominant hemisphere undergoing presurgical MEG assessment at the University of California San Francisco participated in this study (for tumor sites, see Table 1). Twelve individuals were right-handed and two were lefthanded, but all had tumors in the language-dominant hemisphere (13 in the left hemisphere, 1 in the right hemisphere), as defined by the laterality index measured with MEG during picture naming (Findlay et al., 2012) and confirmed by the Wada test (Wada, 1949) when necessary. We note that handedness and hemispheric dominance for language assessed in this way are not necessarily the premorbid ones, as they were determined already in the presence of the tumor. All individuals were native speakers of English. The study was approved by the University of California San Francisco institutional review board, and all participants gave written informed consent.

\section{Materials}

The experimental picture-word interference task was created using 60 colored photographs chosen from the BOSS database (Brodeur, Dionne-Dostie, Montreuil, \& Lepage, 2010) or from the Internet. The photographs belonged to 10 different semantic categories, with six exemplars each (e.g., animals: cow, fish, horse, lion, owl, rabbit). For each photograph, related distractor words were selected from names of the other category-coordinate objects (e.g., pictured cow, distractor "fish"). Unrelated distractors were selected by recombining object names that were semantically and phonologically unrelated to the picture. Thus, all distractor words belonged to the response set. In the neutral condition, a series of five Xs appeared as a distractor. All participants saw each picture once in each condition. Pictures were presented on a white background on the center of the screen. Distractors were presented in black font inside a white box, centered on the picture (see example in Figure 1). The picture-word trials were fully randomized, with one unique list per participant. Participants were instructed to name the picture and to ignore the distractor word. Both speed and accuracy were emphasized.

\section{Procedure}

The presentation of stimuli and the recording of responses were controlled by E-Prime 2.0 software (Psychology Software Tools). Participants were laying down in supine 
Table 1. Characteristics of the Participants

\begin{tabular}{|c|c|c|c|c|c|}
\hline Participant & Confirmed Pathology & Grade & Sex & Age & Overall Error Rate in Present Task \\
\hline 1 & Oligodendroglioma & 2 & $\mathrm{~F}$ & 34 & 6.1 \\
\hline 2 & Diffuse astrocytoma & 2 & $\mathrm{~F}$ & 31 & 5.6 \\
\hline 3 & Glioblastoma & 4 & M & 77 & 3.9 \\
\hline 4 & Anaplastic astrocytoma & 3 & M & 27 & 3.3 \\
\hline 5 & Recurrent anaplastic oligodendroglioma & 3 & $\mathrm{~F}$ & 36 & 3.3 \\
\hline 6 & Oligodendroglioma & 2 & $\mathrm{~F}$ & 35 & 1.7 \\
\hline 7 & Oligodendroglioma & 2 & M & 41 & 1.7 \\
\hline 8 & Diffuse astrocytoma & 2 & M & 51 & 10 \\
\hline 9 & Oligodendroglioma & 2 & M & 58 & 3.9 \\
\hline 10 & Oligodendroglioma & 2 & $\mathrm{~F}$ & 47 & 3.3 \\
\hline 11 & Meningioangiomatosis & $\mathrm{NA}^{* *}$ & $\mathrm{~F}$ & 22 & 2.8 \\
\hline 12 & Recurrent diffuse astrocytoma & 2 & $\mathrm{~F}$ & 37 & 5.6 \\
\hline 13 & Anaplastic astrocytoma & 3 & M & 52 & 1.1 \\
\hline $14^{*}$ & Oligodendroglioma & 2 & $\mathrm{~F}$ & 43 & 6.1 \\
\hline
\end{tabular}

* Indicates the participant with right hemisphere dominance for language. $\mathrm{NA}^{* *}=$ not a tumor; $\mathrm{F}=$ female; $\mathrm{M}=$ male.

position in an electrically and magnetically shielded room, with their heads in the opening of the MEG helmet. Stimuli were projected onto a screen placed above the participants. Vocal responses were recorded with a microphone along the MEG data. Trials began with a fixation cross presented on the center of the screen for a variable duration, between 1.7 and $2.1 \mathrm{sec}$. Then, the picture-word stimulus was presented for 2 sec.

The MEG system (CTF VSM MedTech) contained 275 axial gradiometers. Three localization coils were fixed to the nasion, left, and right preauricular points to monitor the position of participants' heads relative to the gradiometers. The data were low-pass filtered by an antialiasing filter (300 Hz cutoff), digitized at $1200 \mathrm{~Hz}$, and stored for offline analysis. A third order gradiometer configuration was used to reduce noise.

\section{Lesion Analyses}

Lesions were drawn by a trained technician in the native space of participants' T1-weighted or T2-weighted magnetic resonance images (MRIs) and confirmed by a neurologist (R. T. K.). The lesion delineations were subsequently normalized to the Montreal Neurological Institute template and checked again to confirm that no distortions occurred. Percent damage to different areas was determined based on the Automated Anatomical Labeling template in MRIcroN (Rorden, Karnath, \& Bonilha, 2007). We also compared the lesion location of each individual with selected tractography reconstructions of white matter pathways obtained from a group of healthy controls (Rojkova et al., 2016). These analyses allowed us to quantify the proportion of overlap between the lesion's volume and the tracts' volume using Tractotron software as part of the BCBtoolkit (Foulon et al., 2018; www. toolkit.bcblab.com). The selected pathways were chosen based on them passing through the middle temporal gyrus (MTG) given the critical role of this area in language (Sierpowska et al., 2019; Turken \& Dronkers, 2011): the long and posterior segments of the arcuate fasciculus, the inferior frontal occipital fasciculus, and the inferior longitudinal fasciculus. Because of the heterogeneity in lesion distribution, we summarized the lesion profiles using hierarchical clustering over the proportion of damage to areas substantially impacted by the tumor or areas previously associated with word production or picture-word interference, and for the four tracts that pass through the MTG, as mentioned above. The gray matter areas selected for the analysis were inferior temporal gyrus, MTG, including the pole, superior temporal gyrus, including the pole, left inferior frontal gyrus (pars opercularis, pars orbicularis, and pars triangularis), middle frontal gyrus, superior frontal gyrus, insula, and ACC. Clustering techniques group elements such that elements in one same cluster are more similar to each other than to elements in other clusters. Note that the values were selected for the participants' languagedominant hemisphere. The Euclidean distance was used, together with the Ward's criterion. Validation of the cluster solution was achieved via multiscale bootstrap resampling (1000 bootstraps; Suzuki \& Shimodaira, 2006). $p$ Values were derived from the approximately unbiased $p$ value, and we employed an alpha level of .05 . 


\section{Behavioral Analyses}

Vocal responses were examined offline for dysfluent responses or errors: naming the distractor word instead of the picture $(0.2 \%$ of the trials), hesitations $(1.1 \%)$, no responses $(0.4 \%)$, phonological paraphasias $(<0.1 \%)$, semantic paraphasias $(0.5 \%)$, another name rather than the target name (1.1\%), picture not recognized $(0.4 \%)$, and uncategorizable $(0.4 \%)$. The corresponding trials were excluded from all RT and MEG analyses. Naming RTs were calculated manually from the speech signal before trials were separated by condition. Single-trial data and analysis scripts are available via de Open Science Framework (tiny.cc/4q007y). Single-trial RT and accuracy were analyzed with linear and logistic mixed-effects models, respectively Baayen, Davidson, \& Bates, 2008). Models were fitted with the lme 4 package (Version 3.4.4; Bates, Mächler, Bolker, \& Walker, 2015) in R (Version 3.4.3, R Core Team, 2017). Both models included a fixed effect for distractor condition (related, unrelated, neutral; unrelated was the reference) and random slopes for the distractor condition by participant. Single-trial item information was not available. Significance of effects was obtained using the Satterthwaite approximation (lmerTest package Version 3.4.4; Kuznetsova, Brockhoff, \& Christensen, 2017). We also calculated standard (z) scores for each participant based on a jack-knifing approach (i.e., the semantic and lexical effects for a given individual are compared with the group without that individual).

\section{MEG Analyses}

For the MEG data, analyses were performed using FieldTrip (Version 20171231; Oostenveld, Fries, Maris, \& Schoffelen, 2011) in MatlabR2017b. The data were detrended, down-sampled offline to $600 \mathrm{~Hz}$, and segmented into epochs from $0.3 \mathrm{sec}$ prestimulus to $1 \mathrm{sec}$ poststimulus. Before the data were separated by condition, MEG epochs were inspected and excessively noisy sensors were removed. Independent component analysis was then used to correct for artifacts, including eye movements (Jung et al., 2000). Artifact- and error-free data comprised, on average, 56, 55, and 57 trials for the related, unrelated, and neutral conditions, respectively. The signal in single trials was low-pass filtered with a zero-phase shift Butterworth filter with a cutoff frequency of $30 \mathrm{~Hz}$. The data were further segmented from -0.3 to $1 \mathrm{sec}$ before computing the event-related fields (ERFs), calculated by averaging the trials for each condition and participant separately, followed by baseline correction using the averaged activity in the interval of -0.3 to $0 \mathrm{sec}$ relative to picture onset.

Following the evidence that semantic interference (i.e., related vs. unrelated conditions) is mainly associated with electrophysiological differences in the N400 time window and implicates mainly the left temporal lobe (de Zubicaray
\& Piai, 2019), the activity for the related and unrelated conditions was averaged around the N400 time window (i.e., $350-450 \mathrm{msec}$ ) over the left posterior sensors available for all participants. This "N400 activity" was used descriptively to examine the pattern of brain responses over the whole group. For the lexical effect, no information in the literature was available to motivate a specific spatiotemporal dimension of the data. Therefore, this analysis was not conducted for the lexical effect.

In addition, for inferential statistics of the event-related fields, we ran nonparametric cluster-based permutation tests for both semantic and lexical effects (Maris \& Oostenveld, 2007) with no a priori information on sensors or time points (the window of picture onset to $600 \mathrm{msec}$ postonset was examined). Nonparametric cluster-based permutation effectively controls the false alarm rate at the nominal level of 0.05 while comparing the sensors and time points between conditions. The largest cluster in size of adjacent sensors and time points exhibiting a similar difference between the conditions assessed was identified by means of dependent-samples $t$ tests thresholded at an alpha level of .05. The permutation $p$ value was calculated using the Monte Carlo method with 1000 random permutations. A Monte Carlo cluster $p$ value below 5\% (two-tailed testing) was considered significant.

Given the significant results at the sensor level in the N400 time window (see Results section), we then performed source localization of the observed effect to further characterize its spatial distribution using a linearly constrained minimum variance (LCMV) beamforming approach in the time domain (Van Veen, van Drongelen, Yuchtman, \& Suzuki, 1997). The single-trial data were further epoched from -0.3 to $0.45 \mathrm{sec}$ relative to stimulus onset, and the sensor covariance matrix was estimated for the beamforming. The forward model was calculated using a realistically shaped single-shell volume conduction model (Nolte, 2003) based on an MRI template. Ideally, volume conduction models based on the individual patients' MRIs should have been used. However, we could not implement this approach because the segmented and normalized MRIs we obtained were not of sufficient quality for generating 3-D grids of dipole locations that could be averaged over participants. The volume conduction model was then used to compute the lead field matrix, which was done for each participant individually, based on a 3-D grid of dipole locations with equidistant spacing of $10 \mathrm{~mm}$. Thus, individual participants' geometry was considered in the lead-field calculations. In summary, the approach we adopted is sufficient for distinguishing left from right hemisphere sources, as the expected margin of error in localization is smaller than the distance between the left and right hemisphere locations and most of the accuracy errors relate to signal magnitude, rather than location (Vorwerk et al., 2014; Van Uitert, Johnson, \& Zhukov, 2004; Van Den Broek, Reinders, Donderwinkel, \& Peters, 1998). An LCMV beamformer was applied to the whole brain, computing a common (i.e., over all conditions) 
spatial filter for each grid point. The common filter was then applied to the single-trial data from the individual conditions, ensuring that the same spatial filter was used for both conditions. For each dipole location, the source was assumed to have a fixed orientation. To account for the center of the head bias, the Neural Activity Index was used (Van Veen et al., 1997). Finally, the dipole moments were averaged across time within the N400 latency range (i.e., 350-450 msec). The same nonparametric clusterbased permutation approach was used to assess the source-level differences between the two conditions across participants. Given that source reconstruction is a spatial filtering of observations from the scalp and we know from the results of the inferential statistical test the direction of the scalp effect, one-tailed testing was used.

\section{Additional Healthy Control Data}

MEG data from 12 healthy controls (five women, mean age $=60$ years, range $=47-76$ years) performing the same picture-word interference task was also analyzed to allow for a comparison with the patients' data. The materials were 88 colored photographs from the same database as for the main experiment, belonging to 16 different semantic categories with multiple exemplars. These materials largely overlapped with the materials of the main experiment. For each photograph, related and unrelated distractor words were generated in the same way as for the main experiment. The rest of the procedure and apparatus were the same as for the main experiment. The MEG data were preprocessed in the same way as for the main experiment, but the data were segmented into shorter time windows (i.e., -0.3 to $0.6 \mathrm{sec}$ ) as we expected the naming latencies in the healthy control group to be shorter than for the patients and we wanted to avoid including time points already containing speech production ni the ERFs. Following the findings on semantic interference in the patients (see Results section), we ran a nonparametric cluster-based permutation test for the semantic effect in the controls within the N400 time window (i.e., 350-450 msec), including all available left temporal and right temporal sensors. All other parameters of the cluster-based permutation test were identical to that of the patients.

\section{RESULTS}

\section{Lesion Profile}

Figure 2 shows how all 14 participants are grouped in clusters as a function of their gray (left) and white matter (right) lesion profiles. The $y$-axis indicates how dissimilar, according to the Euclidean distance, the individual data points and clusters are from each other. Significant clusters are indicated by the colored outlines. For the gray matter, three different clusters were identified. The lesion overlap of participants pertaining to these three different clusters is shown in Figure 3 (left). Participants 1 and 3 formed one cluster, characterized by lesions overlapping in the inferior temporal gyrus. Participants 2 and 12 formed another cluster, characterized by lesions overlapping in the insula. Participants 5, 6, 7, 8, 9, 10, and 11 formed the third cluster, with inconsistent lesion overlap. Participants 4, 13, and 14 did not enter any clusters, indicating that these three participants have more particular lesions, and are shown separately in Figure 3 (right). For the white matter, one large cluster was identified, including all but Participant 13. Thus, the lesion profile analysis indicates that Participant 13 had a lesion that did not cluster with other participants' lesions both at the gray and white matter levels.

\section{Behavioral Results}

Overall error rates are presented in Table 1 for each participant individually. At the group level, no difference was
Figure 2. Dendrograms of the lesion clusters. Significant clusters are indicated by the colored outlines. (Left) Lesion in gray matter (proportion damage): anterior cingulate, insula, inferior frontal gyrus; pars opercularis, orbitalis, and triangularis; middle frontal gyrus, superior frontal gyrus, inferior temporal gyrus, MTG and pole; and superior temporal gyrus and pole. (Right) Lesion in white matter (proportion damage): arcuate fasciculuslong segment, arcuate fasciculus-posterior segment, inferior frontal occipital fasciculus, inferior longitudinal fasciculus.

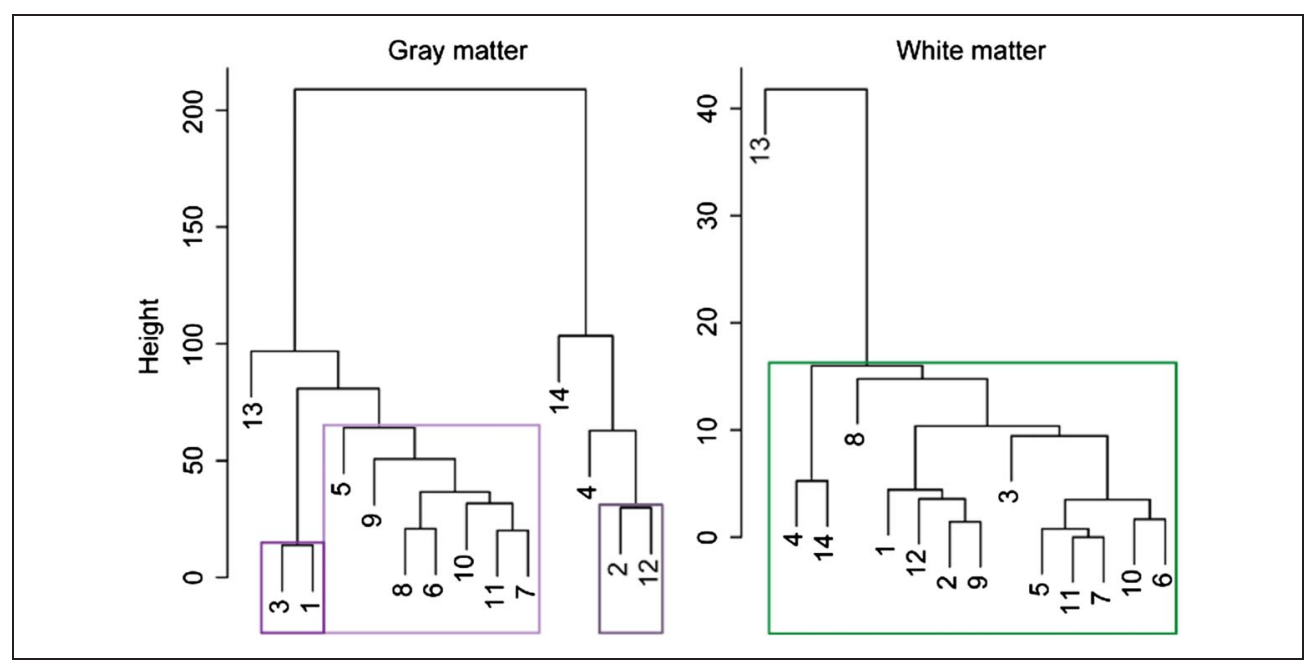




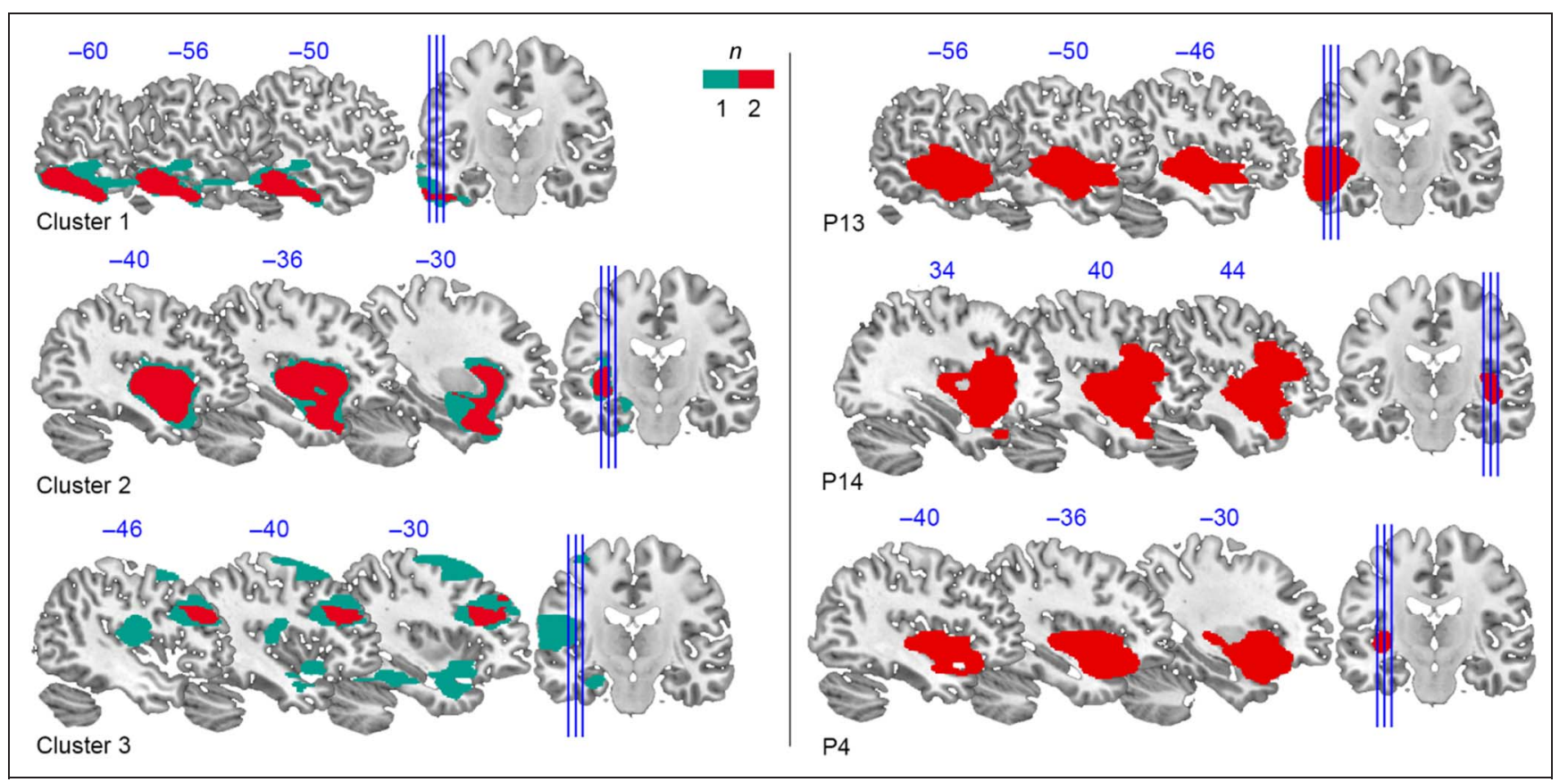

Figure 3. Lesion overlap. (Left) Lesion overlap of the participants for the three identified clusters. The color scale indicates the number of participants for which the overlap consists of. For Clusters 1 and 2, with two participants each, $n=1$ (green) corresponds to $50 \%$ overlap and $n=2$ (red) corresponds to $100 \%$ overlap. For Cluster 3, based on seven participants, $n=1$ (green) corresponds to $14 \%$ overlap and $n=2$ (red) corresponds to $29 \%$ overlap. (Right) Lesion delineation for individual participants not pertaining to any cluster.

found in accuracy between the related and unrelated conditions ( $4.9 \%$ vs. $5.2 \%$, respectively; $b$ estimate $=-0.120$, $S E=0.243, z=0.494, p=.622$ ). More errors were made in the unrelated than in the neutral condition, that is, the lexical interference effect (5.2\% vs. $2.4 \%$, respectively; $b$ estimate $=-0.823, S E=0.273, z=-3.019, p=$ .003 ). Figure 4 (left) shows the RTs for each participant and condition. The median RTs were $1.03 \mathrm{sec}$ for the related condition, $0.978 \mathrm{sec}$ for the unrelated, and 0.913 sec for the neutral. Both lexical and semantic interference effects were found (semantic: $b$ estimate $=0.06, S E=$ $0.01, t=4.66, p<.001$; lexical: $b$ estimate $=-0.06, S E$ $=0.02, t=-2.73, p=.017)$. For the semantic effect, descriptively all participants show semantic interference. For the lexical effect, descriptively three participants showed facilitation (Participants 8, 11, and 12). Figure 4 (middle

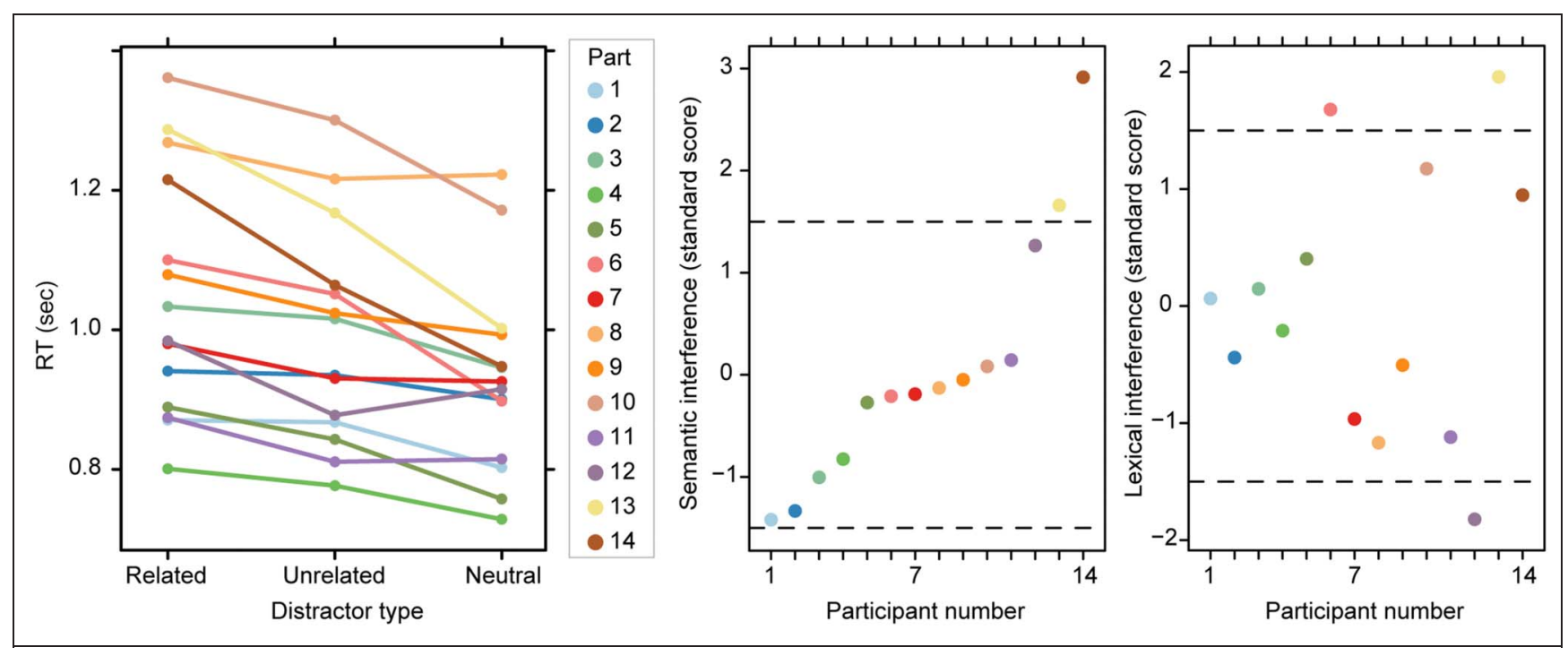

Figure 4. Behavioral results. Each color indicates one participant (Part = participant). (Left) Median RT per participant for each distractor type. (Right) Standardized semantic (left) and lexical (right) interference effects per participant. Each dot represents one participant. Dashed horizontal lines indicate \pm 1.5 standard deviation. 


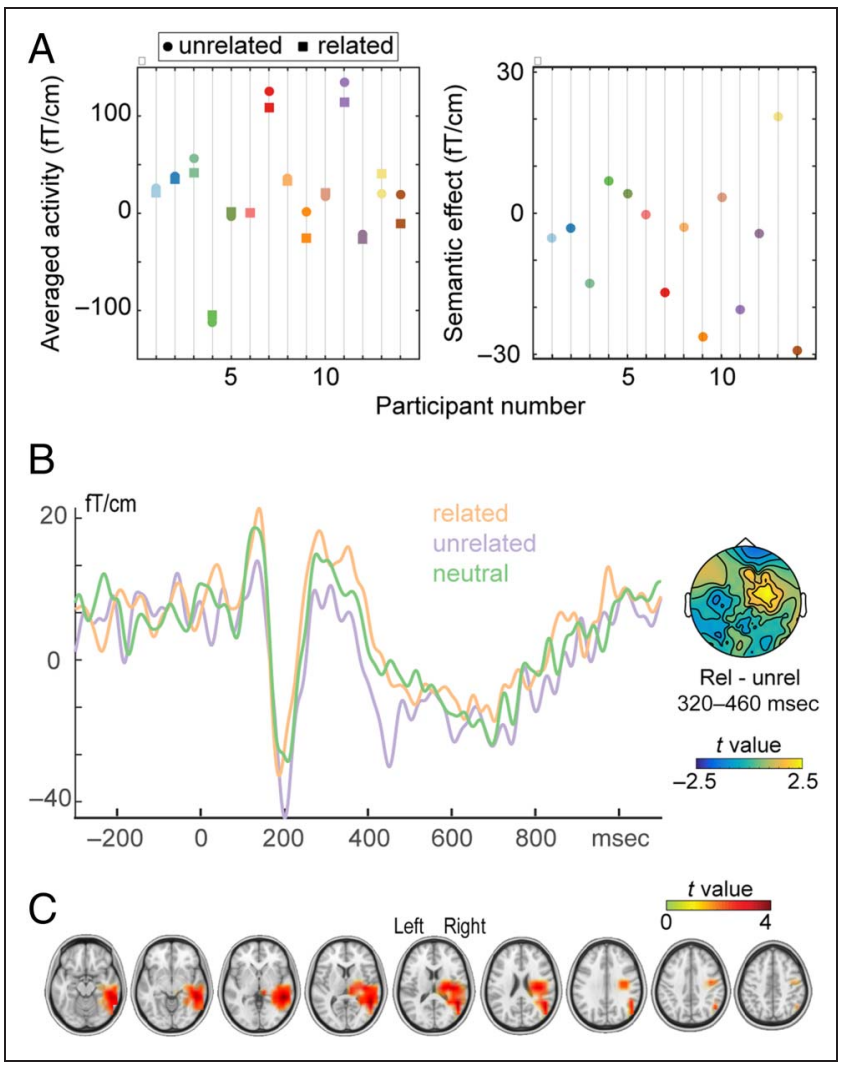

Figure 5. (A) MEG results. Averaged activity between 350 and $450 \mathrm{msec}$ over left posterior sensors for each participant for the unrelated (circle) and related (square) conditions (left) and the semantic effect (related minus unrelated, right). Each dot is one participant. (B) Event-related fields for the related (rel), unrelated (unrel), and neutral conditions for the entire sample averaged over the sensors showing the most pronounced differences for the semantic effect, which can be seen on the right. (C) Source localization on the group level of the semantic effect in the time window $350-450$ msec. Cluster $t$ values are plotted, masked by the statistically significant cluster.

and right) shows the standard score for each participant for both the semantic (middle) and lexical (right) effects. Participants 13 and 14 showed semantic interference effects 1.5 standard deviations larger than the group mean. For the lexical effect, Participants 6 and 13 showed lexical interference effects 1.5 standard deviations smaller than the group mean, whereas Participant 12 showed a lexical facilitation effect 1.5 standard deviations smaller than the group mean. Participant 13 is the only individual to show deviant effects for both semantic and lexical interference following our jack-knifing approach. We note that this individual did not have overall language production problems, as shown, for example, by his high accuracy in picture naming (Table 1). We further tested the abnormality in the scores of Participant 13 for the semantic and lexical effects using a modified paired-samples test appropriate for single cases (Crawford, Howell, \& Garthwaite, 1998). For semantic interference, the effect for Participant 13 was discrepant with the control sample, $t=-5.044$, (estimated percentage of normal population more extreme than Participant $13=0.014 \%$ ). For lexical interference, the effect for Participant 13 was also discrepant with the control sample, but less so than the semantic interference effect, $t=-1.754$ (estimated percentage of normal population more extreme than Participant $13=5.248 \%$ ).

\section{MEG Results}

Figure $5 \mathrm{~A}$ shows the averaged N400 activity between 350 and $450 \mathrm{msec}$ over left posterior sensors for each participant for the related and unrelated conditions (left) and the semantic effect (related minus unrelated, right). Participant 13 presents a deviant pattern over left posterior sensors in the ordering of the conditions compared with the rest of the group, $t=-2.714$ (estimated percentage of normal population more extreme than Participant $13=0.941 \%$; Figure 5A, right).

Regarding the inferential analyses of the MEG eventrelated responses, no significant effects were found for the lexical effect. By contrast, a significant effect was found for the semantic effect (Monte Carlo $p=.010$, two-tailed). Figure 5B shows the ERFs of the entire sample for the three conditions, averaged over the sensors showing the most pronounced differences for the semantic effect. The difference between the related and unrelated conditions was most pronounced in the time window between 320 and $460 \mathrm{msec}$, corresponding to the $\mathrm{N} 400 \mathrm{~m}$ component, over right hemisphere sensors.

We employed a linearly constrained minimum variance beamforming approach in the time domain (Van Veen et al., 1997) to localize the sources of the effect in the 350-450 msec time range. The amplitude of the signal in the 350-450 msec window was significantly different between the two conditions (Monte Carlo $p=.031$, one-tailed). The source localization results are shown in Figure 5C. Cluster $t$ values are plotted, masked by the statistically significant cluster. The source localization indicates that the modulation in signal amplitude in the N400 time window originates in the right hemisphere,

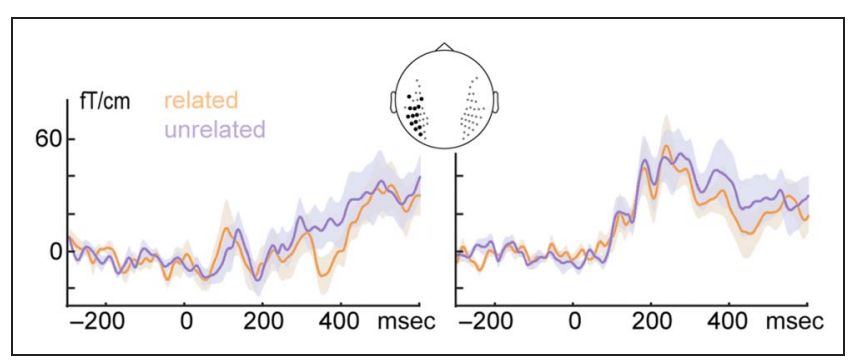

Figure 6. MEG results for the control group. Event-related fields for the related and unrelated conditions over the left temporal sensors associated with the significant cluster (left), indicated by the larger black dots in the scalp map in the middle, and corresponding right temporal sensors (right). The dots indicate the sensors included in the statistical test. The larger black dots indicate the sensors pertaining to the significant cluster. Shaded areas indicate the SEM. 
most prominently in the MTG, but also extending more inferiorly and superiorly. Given that Patient 14 had a brain tumor in the right, language-dominant hemisphere, we repeated the analyses of the ERFs without Patient 14. The pattern of N400 effect with right-lateralized topography in the sample of 13 patients with tumors in the left language-dominant hemisphere was virtually identical to the pattern shown in Figure 5B (Figure S1). We also repeated the analysis with the 12 right-handed patients. The pattern of N400 effect with right-lateralized topography was again present in the sample of 12 patients (Figure S1). This finding underscores that the right hemisphere shift observed in the whole group is not driven by the individual with right hemisphere language dominance or by the two left-handed individuals.

For the healthy controls, a significant cluster was identified for the semantic effect between 350 and $450 \mathrm{msec}$ (Monte Carlo $p=.046$, two-tailed). Figure 6 shows the ERFs for the related and unrelated conditions, averaged over the left temporal sensors associated with the significant cluster (left panel; and dark dots in the scalp map) and right temporal (right panel) sensors (tested sensors are shown as dots in Figure 6). The significant difference between the related and unrelated conditions was only found over left temporal sensors, indicated by the black dots in Figure 6. This finding is in contrast with the results of the patient group, for whom the significant cluster was observed over right sensors only, as also confirmed by the source localization results. Despite the different morphology of the ERFs between the patients and the controls, in both cases, the amplitude in the unrelated condition deviates most from zero (i.e., baseline), whereas the related condition is closest to the baseline amplitude values.

\section{DISCUSSION}

We assessed the feasibility of administering picture-word interference during MEG recordings in presurgical brain tumor patients and, more importantly, examined tumorinduced neuronal reorganization. On the group level, we observed the expected lexical interference and semantic interference effects in the picture naming times and the N400-like event-related responses associated with the semantic effect. The N400 semantic effect had sources in the right temporal cortex in tumor patients in contrast to the left hemisphere N400 effect typically found in healthy controls (de Zubicaray \& Piai, 2019; Piai et al., 2014). One participant with a lesion affecting the left temporal lobe and underlying white matter tracts showed a deviant pattern in behavior as well as in N400 event-related responses. We discuss each of these effects below.

We observed the expected lexical interference and semantic interference effects in the picture-naming times and in the error rates for the lexical interference effect. This is in line with previous research (Piai et al., 2016;
Damian \& Bowers, 2003; Roelofs, 2003; La Heij, 1988; Glaser \& Düngelhoff, 1984), including the finding that a semantic interference effect in the error rates is not typically observed (e.g., Piai \& Knight, 2018).

Regarding the MEG analyses, for the semantic effect, a difference was found at the group level between the related and unrelated conditions in the expected time window (de Zubicaray \& Piai, 2019), but with an altered topographical distribution. The effect was shifted to the right, as seen over the scalp, which was also confirmed by the source localization analysis, suggesting involvement of the right hemisphere at the group level. By contrast, for a group of healthy controls, the semantic effect in the 350-450 msec time window was only significant over left temporal, but not right temporal, sensors.

Evidence has accumulated for the right hemisphere's role in language in the case of brain tumors in the language-dominant left hemisphere. The involvement of the right hemisphere has been found postoperatively following resection of the left hemisphere, for example, using MEG (Traut et al., 2019). A number of studies has also identified right-hemisphere involvement in language function preoperatively (De Witte et al., 2014; Rösler et al., 2014; Krieg et al., 2013; Thiel et al., 2005), a pattern of reorganization likely induced by the tumor in the left hemisphere. The same explanation is likely for the present findings. Altogether, these findings indicate that tumor-induced reorganization of function, even before surgery, is a true phenomenon (Duffau, 2014) that needs to be taken into account when studying which patterns of lesion will lead to dysfunctions pre- and postoperatively. This pattern of reorganization may explain why most individuals do not present with substantial language deficits despite the lesions in the dominant language hemisphere. It is also possible that individuals with more right-hemisphere reorganization presurgically will show less severe deficits following surgery to the left hemisphere.

Importantly, by using electrophysiology, we show that the pattern of activity found in the right hemisphere resembles the brain responses usually found over the left hemisphere in healthy individuals both in timing and amplitude modulation as a function of the task (de Zubicaray \& Piai, 2019; Piai et al., 2014). This is an advantage of using electrophysiological techniques over other techniques to understand lesion-dependent language deficits and plasticity. Language-related processes happen at a fast timescale and are reflected in the timespecific amplitude modulations of brain responses. If a particular neural signature is well characterized, as in the case of the N400 response (Kutas \& Federmeier, 2011), it can be used to examine whether processes are supported by "new", otherwise atypical areas (e.g., shifted in hemisphere) due to a brain lesion. This approach provides important insights for understanding neuroplasticity, as it indicates not only that "new" areas are involved but also better characterizes "what" these 
new areas are doing (see for a similar argument Piai et al., 2017).

The use of a challenging naming task enabled us to observe relatively normal performance in word production by the patients along with the involvement of "atypical" (right hemisphere homologue) brain areas. Although most patients do not present with language deficits when assessed objectively, patients do report experiencing fatigue during every day language use. It may be the case that these atypical areas are sufficient for overcoming impairment, but not as efficient for (some) language tasks, explaining patients' subjective experience. These are important avenues for future research.

By using a jack-knifing approach, we identified two participants performing more poorly than 1.5 standard deviations from the group's mean with respect to the semantic interference effect and to the lexical interference effect. One participant (Participant 13) showed poorer performance for both effects. The analyses on the profile of the lesions identified different clusters at the gray and white matter levels. At the gray matter level, three participants showed a more distinct lesion profile, not entering any clusters (including Participant 13). By contrast, at the white matter level, all participants, with the exception of Participant 13, were clustered together, indicating that Participant 13 had a unique white matter lesion pattern. The planned MEG analyses for the semantic effect (de Zubicaray \& Piai, 2019) also identified a pattern of activity over left posterior sensors that was different for Participant 13. The discrepant patterns observed for this participant were confirmed by statistical analyses appropriate for single-participant comparisons to a control group (Crawford et al., 1998).

Some specific disease characteristics may be able to explain the observed pattern. Participant 13 had a fastgrowing type of tumor, Grade III anaplastic astrocytoma. Whereas Participants 3 and 4 also had high-grade tumors, only in Participant 13 did the tumor infiltrate large parts of the language-dominant temporal lobe. In particular, this was the only individual with such a large portion of MTG involvement, with damage in all ventral and dorsal tracts inspected. The combination of a fast-growing tumor, which limits the time for functional reorganization (Kong, Gibb, \& Tate, 2016; Desmurget, Bonnetblanc, \& Duffau, 2007), in this critical location, that is, the language-dominant MTG and the fibers passing through it (Piai \& Knight, 2018; Griffis, Nenert, Allendorfer, \& Szaflarski, 2017; Turken \& Dronkers, 2011; Schwartz et al., 2009; Dronkers, Wilkins, Van Valin, Redfern, \& Jaeger, 2004), could potentially explain the deficits observed across both lexical and semantic effects.

One limitation of this study is that the distribution of the lesions in the present sample was heterogeneous, but this heterogeneity was in fact helpful for identifying a possible lesion-symptom relationship. Another limitation of this study is that the number of trials was not sufficient for analyzing the MEG responses as a function of experimental condition at the single-participant level. A third limitation is the approach we used for the source localization analyses. Ideally, individual MRIs should have been used for the volume conductors, including the modeling of the tumors. However, estimating the conductivity of the tumor is not a trivial task, given the uncertainty in the conductivity values and the variable degree of vascularization across patients. In this case, modeling the tumor with inaccuracies in the conductivity values may be more detrimental than the inaccuracies introduced by not modeling the tumor (e.g., Van Den Broek et al., 1998). Nonetheless, there is no reason to expect that the presence of the tumor in the left hemisphere would affect the source reconstruction results, such that a right hemisphere shift of the amplitude difference between the two conditions would be produced as an artifact (Vorwerk et al., 2014). Moreover, the location of the strongest sources of the amplitude differences for the semantic effect is not random, but rather in the right hemisphere homologue of where most of the sources generating the N400 effect are located (Lau et al., 2008). It is more plausible that the amplitude differences observed are indeed generated in the right hemisphere. This issue remains an important one for our field, and future studies with detailed volume conduction modeling are needed to confirm our findings.

\section{Conclusions}

The replication of the classic lexical and semantic interference effects behaviorally and of the MEG semantic effect attest to the reliability and validity of the approach. The present results provide support for neuroplasticity in the presurgical phase, with the right hemisphere performing similar neuronal computations (reflected in MEG N400 event-related responses) as the left hemisphere typically performs (see also Piai et al., 2017). Additionally, we identified word production deficits in one participant with a unique lesion profile, also affecting the $\mathrm{N} 400 \mathrm{~m}$ pattern of results. An important question for future research is whether the behavioral and/or electrophysiological patterns observed presurgically with such a challenging word production task are predictive of an individual's deficits intraoperatively and recovery postoperatively.

\section{Acknowledgments}

This study was supported by a gravitation grant of the Language in Interaction Consortium (024.001.006) from the Netherlands Organization for Scientific Research and by a Rubicon grant (446-13-009) and a Veni grant (451-17-003) to V.P. from the Netherlands Organization for Scientific Research. The funders had no role in the design, analyses, and report of this study. The authors are indebted to the participating patients. The authors would also like to thank Robert Oostenveld and Maria Carla Piastra for helpful discussions. 
Reprint requests should be sent to Vitória Piai, Radboud University, Montessorilaan 3, 6525HR Nijmegen, the Netherlands, or via e-mail: v.piai@donders.ru.nl.

\section{REFERENCES}

Anderson, S. W., Damasio, H., \& Tranel, D. (1990). Neuropsychological impairments associated with lesions caused by tumor or stroke. Archives of Neurology, 47, 397-405.

Baayen, R. H., Davidson, D. J., \& Bates, D. M. (2008). Mixedeffects modeling with crossed random effects for subjects and items. Journal of Memory and Language, 59, 390-412.

Bates, D., Mächler, M., Bolker, B., \& Walker, S. (2015). Fitting linear mixed-effects models using lme4. Journal of Statistical Software, 67, 1-48.

Brodeur, M. B., Dionne-Dostie, E., Montreuil, T., \& Lepage, M. (2010). The bank of standardized stimuli (BOSS), a new set of 480 normative photos of objects to be used as visual stimuli in cognitive research. PLoS One, 5, e10773.

Brownsett, S. L. E., Ramajoo, K., Copland, D., McMahon, K. L., Robinson, G., Drummond, K., et al. (2019). Language deficits following dominant hemisphere tumour resection are significantly underestimated by syndrome-based aphasia assessments. Aphasiology, 33, 1163-1181.

Crawford, J. R., Howell, D. C., \& Garthwaite, P. H. (1998). Payne and Jones revisited: Estimating the abnormality of test score differences using a modified paired samples t test. Journal of Clinical and Experimental Neuropsychology, 20, 898-905.

Damian, M. F., \& Bowers, J. S. (2003). Locus of semantic interference in picture-word interference tasks. Psychonomic Bulletin \& Review, 10, 111-117.

de Zubicaray, G. I., \& Piai, V. (2019). Investigating the spatial and temporal components of speechproduction. In G. I. de Zubicaray \& N. O. Schiller (Eds.), The Oxford handbook of neurolinguistics. Oxford, United Kingdom: Oxford University Press.

De Witte, E., Satoer, D., Robert, E., Colle, H., Verheyen, S., Visch-Brink, E., et al. (2015). The Dutch linguistic intraoperative protocol: A valid linguistic approach to awake brain surgery. Brain and Language, 140, 35-48.

De Witte, E., Van Hecke, W., Dua, G., De Surgeloose, D., Moens, M., \& Mariën, P. (2014). Atypical cerebral language dominance in a right-handed patient: An anatomoclinical study. Clinical Neurology and Neurosurgery, 117, 12-21.

Desmurget, M., Bonnetblanc, F., \& Duffau, H. (2007). Contrasting acute and slow-growing lesions: A new door to brain plasticity. Brain, 130, 898-914.

Dronkers, N. F., Wilkins, D. P., Van Valin, R. D., Redfern, B. B., \& Jaeger, J. J. (2004). Lesion analysis of the brain areas involved in language comprehension. Cognition, 92, 145-177.

Duffau, H. (2007). Contribution of cortical and subcortical electrostimulation in brain glioma surgery: Methodological and functional considerations. Clinical Neurophysiology, 37, 373-382.

Duffau, H. (2014). The huge plastic potential of adult brain and the role of connectomics: New insights provided by serial mappings in glioma surgery. Cortex, 58, 325-337.

Findlay, A. M., Ambrose, J. B., Cahn-Weiner, D. A., Houde, J. F., Honma, S., Hinkley, L. B., et al. (2012). Dynamics of hemispheric dominance for language assessed by magnetoencephalographic imaging. Annals of Neurology, 71, 668-686.

Foulon, C., Cerliani, L., Kinkingnéhun, S., Levy, R., Rosso, C., Urbanski, M., et al. (2018). Advanced lesion symptom mapping analyses and implementation as BCBtoolkit. GigaScience, 7, 1-17.
Glaser, W. R., \& Düngelhoff, F. J. (1984). The time course of picture-word interference. Journal of Experimental Psychology: Human Perception and Performance, 10, 640-654

Griffis, J. C., Nenert, R., Allendorfer, J. B., \& Szaflarski, J. P. (2017). Damage to white matter bottlenecks contributes to language impairments after left hemispheric stroke. Neuroimage: Clinical, 14, 552-565.

Jung, T. P., Makeig, S., Humphries, C., Lee, T. W., McKeown, M.J., Iragui, V., etal. (2000). Removing electroencephalographic artifacts by blind source separation. Psychophysiology, 37, $163-178$.

Kong, N. W., Gibb, W. R., \& Tate, M. C. (2016). Neuroplasticity: Insights from patients harboring gliomas. Neural Plasticity, 2016, 12.

Krieg, S. M., Sollmann, N., Hauck, T., Ille, S., Foerschler, A., Meyer, B., et al. (2013). Functional language shift to the right hemisphere in patients with language-eloquent brain tumors. PLoS One, 8, e75403.

Kutas, M., \& Federmeier, K. D. (2011). Thirty years and counting: Finding meaning in the N400 component of the event-related brain potential (ERP). Annual Review of Psychology, 62, 621-647.

Kuznetsova, A., Brockhoff, P. B., \& Christensen, R. H. B. (2017). lmerTest package: Tests in linear mixed effects models. Journal of Statistical Software, 82, 1-26.

La Heij, W. (1988). Components of stroop-like interference in picture naming. Memory and Cognition, 16, 400-410.

Lau, E. F., Phillips, C., \& Poeppel, D. (2008). A cortical network for semantics: (De)constructing the N400. Nature Reviews Neuroscience, 9, 920-933.

Maris, E., \& Oostenveld, R. (2007). Nonparametric statistical testing of EEG- and MEG-data. Journal of Neuroscience Methods, 164, 177-190.

Nolte, G. (2003). The magnetic lead field theorem in the quasistatic approximation and its use for magnetoencephalography forward calculation in realistic volume conductors. Pbysics in Medicine and Biology, 48, 3637-3652.

Oostenveld, R., Fries, P., Maris, E., \& Schoffelen, J.-M. (2011). FieldTrip: Open source software for advanced analysis of MEG, EEG, and invasive electrophysiological data. Computational Intelligence and Neuroscience, 2011, 9.

Piai, V., \& Knight, R. T. (2018). Lexical selection with competing distractors: Evidence from left temporal lobe lesions. Psychonomic Bulletin \& Review, 25, 710-717.

Piai, V., Meyer, L., Dronkers, N. F., \& Knight, R. T. (2017) Neuroplasticity of language in left-hemisphere stroke: Evidence linking subsecond electrophysiology and structural connections. Human Brain Mapping, 38, 3151-3162.

Piai, V., Riès, S. K., \& Swick, D. (2016). Lesions to lateral prefrontal cortex impair lexical interference control in word production. Frontiers in Human Neuroscience, 9, 721.

Piai, V., Roelofs, A., Jensen, O., Schoffelen, J.-M., \& Bonnefond, M. (2014). Distinct patterns of brain activity characterise lexical activation and competition in spoken word production. PLoS One, 9, e88674.

R Core Team. (2017). R: A language and environment for statistical computing. R Foundation for Statistical Computing. Vienna. Retrieved from https://www.r-project.org.

Roelofs, A. (2003). Goal-referenced selection of verbal action: Modeling attentional control in the stroop task. Psychological Review, 110, 88-125.

Roelofs, A., \& Piai, V. (2011). Attention demands of spoken word planning: A review. Frontiers in Psychology, 2, 307 .

Rofes, A., \& Miceli, G. (2014). Language mapping with verbs and sentences in awake surgery: A review. Neuropsychology Review, 24, 185-199. 
Rojkova, K., Volle, E., Urbanski, M., Humbert, F., Dell'Acqua, F., \& Thiebaut de Schotten, M. (2016). Atlasing the frontal lobe connections and their variability due to age and education: A spherical deconvolution tractography study. Brain Structure and Function, 221, 1751-1766.

Rorden, C., Karnath, H.-O., \& Bonilha, L. (2007). Improving lesion-symptom mapping. Journal of Cognitive Neuroscience, 19, 1081-1088.

Rösler, J., Niraula, B., Strack, V., Zdunczyk, A., Schilt, S., Savolainen, P., et al. (2014). Language mapping in healthy volunteers and brain tumor patients with a novel navigated TMS system: Evidence of tumor-induced plasticity. Clinical Neurophysiology, 125, 526-536.

Satoer, D., Vincent, A., Smits, M., Dirven, C., \& Visch-brink, E. (2013). Spontaneous speech of patients with gliomas in eloquent areas before and early after surgery. Acta Neurochirurgica, 155, 685-692.

Satoer, D., Visch-Brink, E., Dirven, C., \& Vincent, A. (2016). Glioma surgery in eloquent areas: Can we preserve cognition? Acta Neurochirurgica, 158, 35-50.

Schwartz, M. F., Kimberg, D. Y., Walker, G. M., Faseyitan, O., Brecher, A., Dell, G. S., et al. (2009). Anterior temporal involvement in semantic word retrieval: Voxel-based lesionsymptom mapping evidence from aphasia. Brain, 132, 3411-3427.

Sierpowska, J., Gabarrós, A., Fernandez-Coello, A., Camins, À., Castañer, S., Juncadella, M., et al. (2017). Words are not enough: Nonword repetition as an indicator of arcuate fasciculus integrity during brain tumor resection. Journal of Neurosurgery, 126, 435-445.

Sierpowska, J., Gabarrós, A., Fernández-Coello, A., Camins, À., Castañer, S., Juncadella, M., et al. (2019). White matter pathways and semantic processing: Intrasurgical and lesionsymptom mapping evidence. Neuroimage: Clinical, 22, 101704 .
Suzuki, R., \& Shimodaira, H. (2006). Pvclust: An R package for assessing the uncertainty in hierarchical clustering. Bioinformatics, 22, 1540-1542.

Thiel, A., Habedank, B., Winhuisen, L., Herholz, K., Kessler, J., Haupt, W. F., et al. (2005). Essential language function of the right hemisphere in brain tumor patients. Annals of Neurology, 57, 128-131.

Traut, T., Sardesh, N., Bulubas, L., Findlay, A., Honma, S. M., Mizuiri, D., et al. (2019). MEG imaging of recurrent gliomas reveals functional plasticity of hemispheric language specialization. Human Brain Mapping, 40, 1082-1092.

Turken, A. U., \& Dronkers, N. F. (2011). The neural architecture of the language comprehension network: Converging evidence from lesion and connectivity analyses. Frontiers in Systems Neuroscience, 5, 1.

Van Den Broek, S. P., Reinders, F., Donderwinkel, M., \& Peters, M. J. (1998). Volume conduction effects in EEG and MEG. Electroencephalography and Clinical Neurophysiology, 106, 522-534.

Van Uitert, R., Johnson, C., \& Zhukov, L. (2004). Influence of head tissue conductivity in forward and inverse magnetoencephalographic simulations using realistic head models. IEEE Transactions on Biomedical Engineering, 51, 2129-2137.

Van Veen, B. D., van Drongelen, W., Yuchtman, M., \& Suzuki, A. (1997). Localization of brain electrical activity via linearly constrained minimum variance spatial filtering. IEEE Transactions on Biomedical Engineering, 44, 867-880.

Vorwerk, J., Cho, J. H., Rampp, S., Hamer, H., Knösche, T. R., \& Wolters, C. H. (2014). A guideline for head volume conductor modeling in EEG and MEG. Neuroimage, 100, 590-607.

Wada, J. (1949). A new method of determining the side of cerebral speech dominance: A preliminary report on the intracarotid injection of sodium amytal in man. Igaku to Seibutsugaku, 14, 221-222. 